\title{
ROLE OF EPITHELIAL MESENCHYMALTRANSITION IN HEPATIC FIBROGENESIS \\ By
}

MONA K. ZOHEIRY and EMAN G. EL-AHWANY*

Department of Immunology, Theodor Bilharz Research Institute, P.O. Box 30 , Imbaba, 12411, Egypt ('Correspondence: ahwany@aucegypt.edu)

\begin{abstract}
Liver fibrosis is a gradual process of increased secretion and decreased degradation of extracellular materials. Two cell types are now well recognized as being involved in liver fibrosis, i.e. hepatic stellate cells (HSCs) and portal mesenchymal cells. This process is initiated by the damage of hepatic cells, which leads to activation of hepatic stellate cells that differentiate into myofibroblasts leading to the formation of liver fibrosis. On the other hand, the epithelialmesenchymal transition and mesenchymal-epithelial transition are crucial for the regulation of cellular plasticity during liver fibrosis. The EMT is a process in which molecular reprogramming leads epithelial cells to adopt a mesenchymal phenotype. During EMT, epithelial cells gain mesenchymal features which include changes in the expression of epithelial markers. The EMT process plays fundamental roles during embryogenesis, tissue fibrosis, and carcinogenesis. As multiple experimental studies of liver fibrosis have confirmed that established liver fibrosis is reversible upon cessation of the causative agent, modulation of the EMT markers could be promising as potential therapeutic agents. Better understanding of the molecular cascades of intracellular fibrogenic signaling and genetic factors that controlling the expression of EMT markers would be a powerful strategy for early diagnosis and treatment liver fibrosis at the genetic level. Activating or silencing of the responsible genes may be an efficient and more specific approach for treating liver fibrosis either through the arrest of EMT or the induction of MET.
\end{abstract}

Keywords: Liver fibrosis; epithelial-mesenchymal transition; TGF- $\beta$; Extracellular matrix; miRNA

\section{Introduction}

Liver fibrosis is characterized by the excessive deposition of ECM involving molecular and histological re-arrangement of various types of collagens, proteoglycans as hyaluronic acid and structural glycoproteins (Gressner et al, 2009; Kumar and Mahato, 2015). It is considered as a wound healing scar response following acute and chronic liver diseases including chronic hepatitis B \& $\mathrm{C}$, autoimmune hepatitis, non-alcoholic steatohepatitis and alcoholic liver disease (Friedman, 2008). The patho-histological findings of liver cirrhosis, the end-stage of liver fibrosis, show hepatocellular death, a lobular inflammatory cell infiltrate excessive deposition of ECM proteins and the appearance of regenerative nodules that may result in liver failure, portal hypertension and hepatocellular carcinoma (Friedman, 2008). In addition, liver fibrosis is highly associated with chronic hepatocellular injury and subsequent inflammatory response that produc- es inflammatory cytokines and recruits inflammatory leukocytes into the injured site. This inflammatory circumstance in the liver drives the activation of HSCs through various fibrogenic mediators including transforming growth factor-beta (TGF- $\beta$ ) and PDGF (Friedman, 2008; Gressner et al, 2009). The pathophysiology of liver injury has attracted the interest of experimentalists and clinicians over many centuries (Pinzani and Macias-Barragan, 2010). With the discovery of liver- HSC, the insight into the cellular and molecular pathobiology of liver fibrosis has evolved and the pivotal role of HSC as a precursor cell-type for ECMproducing myofibroblasts were established (Hernandez-Gea and Friedman, 2011). Although activation and trans-differentiation of HSC to myofibroblasts is still regarded as the pathogenetic key mechanism of fibrogenesis, recent studies point to a prominent heterogeneity of the origin of myofibroblasts (Pinzani and Macias-Barragan, 2010). Liver 
fibrosis starts with injury, necrosis or apoptosis of hepatocytes and inflammationconnected activation of hepatic stellate cells (HSC) triggering their trans-differentiation to myofibroblasts (Roy et al, 2015). New pathogenetic mechanisms concern: (i) the influx of bone marrow-derived cells (Fibrocytes); (ii) circulating monocytes and (iii) epithelial-mesenchymal transition (EMT) of epethelial cells of bile duct and hepatocytes. These mechanisms enlarge the pool of matrix-synthesizing myofibroblasts in the damaged liver (Gressner et al, 2009). Disturbance of the balance between collagen synthesis \& degradation led to extracellular accumulation and liver fibrosis (Gressener et al, 2007; Friedman, 2008).

Epithelial-Mesenchymal Transition: the understanding of fibrosis has been strikingly enhanced by the characterization of cellular effectors, key inflammatory/pro-fibrogenic cytokines and molecular mediators and by the emergence of new pathogenetic scenarios (Ding and Chen, 2012). A major determinant of fibrosis is the continuous expansion of fibroblasts/myofibroblasts that prompts the question of how this cellular pool can be fed. EMT, by generating new mesenchymal cells, may contribute substantially to the increase of interstitial fibroblasts/ myofibroblasts and have an important role in the pathogenesis of liver fibrosis (Lorenzini et $a l, 2010)$. EMT also can promote the progression of fibrotic disease by generating new mesenchymal cells, which can expand the pool of interstitial fibroblasts (Lee et al, 2012). It is estimated that in kidney fibrosis, $66 \%$ of fibroblasts are the result of EMT, in heart the number claims to about $20 \%$. Very recent studies claim that $40 \%$ of hepatic fibrosis derived from hepatocytes, but these data need further confirmation (Gressner and Gao, 2014).

EMT involves a series of changes through which epithelial cells lose their epithelial characteristics and acquire properties of mesenchymal cells. EMT facilitates cell movement and the generation of new tissue types and also contributes to the pathogenesis of disease (Lee et al, 2012). EMT may be classified into three subtypes. Type 1 EMT: takes place during embryonic development and organogenesis. Type 2 EMT: involves transition of secondary epithelial cells to resident tissue fibroblasts and is associated with wound healing, tissue regeneration, and organ fibrosis. In contrast to type 1 , type 2 EMT is induced in response to inflammation, but stops once inflammation is attenuated, especially during wound healing and tissue regeneration (Lo' pez-Novoa and Nieto, 2009) Type 3 EMT: occurs in carcinoma cells that have formed solid tumors and is associated with their transition to metastatic tumor cells. Hepatic fibrosis is good example of type 2 EMT (Lo'pez-Novoa and Nieto, 2009). These different EMT programs may be induced and regulated by a common set of stimuli, signal transduction pathways, transcription factors, and posttranslational regulations (Zeisberg and Neilson, 2009).

1-Mechanism of EMT: Two key changes occurring in epithelial cells undergoing EMT are detachment from neighboring epithelial cells and the migration into the interstitium where they may start producing matrix (Acloque et al, 2009). The molecular hallmarks of EMT include E-cadherin downregulation, responsible for the loss of cellcell adhesion and the subsequent detachment from the parent epithelium; up-regulation of matrix-degrading proteases that digest epithelial basement membrane; up-regulation and/or nuclear translocation of transcription factors underlying the specific gene program of EMT such as $\beta$-catenin, Smads and members of the Snail family; denovo expression of mesenchymal proteins such as fibroblast specific protein 1 (FSP1) and $\alpha$-SMA; Ras (renin angiostensin system) homologous (Rho) guanosinetriphosphatase (GTPase)mediated cytoskeletal reorganization to favor cell shape changes and to activate motility; loss of cytokeratin and other epithelial-associated markers; and finally, the production of interstitial-type matrix compo- 
nents such as collagen types I \& III and fibronectin (Thiery and Sleeman, 2006; Guarino et al, 2009).

The factors acting on cell surface receptors seem to play important roles in accomplishing EMT (Yusuf and Brand-Saberi, 2006) Matrix-degrading proteases, include MMPs, released from damaged epithelial cells, inflammatory cells, or activated myofibroblasts possibly initiate the process by dismantling the epithelial basement membrane. TGF- $\beta$ is also known to up-regulate matrixdegrading MMPs, and thus, it could participate in basement membrane breakdown (Xue et al, 2013). The dissolution of the basement membrane may result in direct contact between epithelial cells and type I collagen-rich stromal microenvironment, which under normal conditions is prevented by an intact basement membrane. Exposure to interstitial type I collagen, which epithelial cells do not come into contact with in normal conditions, as well as to high concentrations of TGF- $\beta$ and other growth factors present in the interstitial stroma destabilize the epithelial phenotype and encourage EMT (Keeley et al, 2011). Stimulation of integrin-linked kinase (ILK) or $\beta$-catenin pathway and activation of the Rho GTPases could provide additional signals, eliciting the completion of cell dissociation and inducing the cytoskeleton remodeling needed for cell reshaping and movement (Guarino et $a l, 2009)$. Transitioning cells liberated from contact with neighbors and fully equipped for migration, can leave the epithelium and translocate into the surrounding interstitium where they are supported by local stimuli. Looking like true mesenchymal cells, they can be initiated to produce new matrix.

2. Molecular events in EMT: 2.1. Central effector Snail: In most experimental systems, EMT takes place by cytokine/growth factor-driven or matrix- driven stimulation of cell surface receptors (Fig. 1) or by activation of their downstream signaling mediators (Guarino et al, 2009). All lead ultimately to increased activity of the nuclear tran- scription factor Snail, which acts as a master organizer of EMT by repressing the epithelial genes and activating the mesenchymal ones (Peinado et al, 2007). Snail is negatively regulated by glycogen synthase kinase- $3 \beta$ $(\mathrm{GSK}-3 \beta)$, which in resting epithelial cells is maintained in an active state to promote $\mathrm{Sn}$ ail's nuclear export and its cytoplasmic degradation thereby preventing EMT. In an opposite fashion, Snail is positively regulated by lisyl oxidases (LOXs) that, instead, stabilize Snail thereby favoring EMT (Yusuf and Brand-Saberi, 2006; Guarino et al, 2009).

2.2. EMT signaling pathways: a. Transforming growth factor (TGF)- $\beta$. It is an important cytokine for the induction of the EMT in liver fibrosis (Bi et al, 2012). EMT of hepatocytes is dependent on the balance between apoptotic and survival mechanisms. The process of EMT requires the action of MMTs \& a TGF- $\beta$ dependent snail-mediated down-regulation of E-cadherin both contributing to the release of epithelial cells from cell-cell and cell-basement membrane binding (Jou and Diehl, 2010).

The BMP-7, a member of TGF- $\beta$ superfamily, does not only inhibit EMT, but even induce a mesenchymal-epithelial (retro-) transition (reverse EMT $=$ MET) (Sugimoto et al, 2007). BMP-7 has been shown to inhibit TGF- $\beta$ dependent EMT of hepatocytes and the progression of experimental fibrosis in mice (Kisselva et al, 2006). It has, also, anti-apoptotic properties, anti-inflammatory, and proliferation-stimulating effects (Wang and Hirschberg, 2004). BMP-7 inhibits TGF- $\beta$ signaling via Smads (Ten Dijke and Hill, 2004; Neilson, 2005), which transduce the effect of the latter cytokine from its receptor, a serine/threonine kinase, to Smadbinding element of respective target genes in the nucleus. Also, several trapping proteins such as the small proteoglycans decorin and biglycan, latency associated peptide (LAP), Bambi, KCP (kielinchordin-like protein), gremlin, and alpha-2-macroglobulin change the balance between TGF- $\beta$ and BMP-7 in favor of an anti-EMT effect by binding and 
neutralization of TGF- $\beta$ (Diaz et al, 2008). Similarly, the important downstream-modulator protein connective tissue growth factor (CTGF/CCN2) (Diaz et al, 2008), which is expressed in hepatocytes, HSC, portal fibroblasts, and cholangiocytes (Ten Dijke and Hill, 2004), changes the functional TGF- $\beta$ /BMP-7 ratio (Friedman, 2008). CTGF is over-expressed in experimental and human liver cirrhosis mediated mainly by TGF- $\beta$, but also by ET-1,TNF- $\alpha$, VEGF, nitrogen oxide (NO), prostaglandin E2, thrombin, high glucose, and hypoxia (Gressener et al, 2008), CTGF inhibits BMP, but activates TGF- $\beta$ signaling by modulation of the receptor-binding of these ligands (Jou and Diehl, 2010). The prominent functional role of CTGF is supported by recent data, which show sustained anti-fibrotic effects if CTGF expression is reduced by siRNA (Hernandez-Gea and Friedman, 2011).

The signaling generated by TGF- $\beta$ is mediated by type I \& II trans-membrane serinethreonine kinase receptors and is initiated by binding of the extracellular TGF- $\beta$ ligand to the type II receptor, which recruits the type I receptor into a heteromeric complex where it is phosphorylated by the type II receptor, resulting in its activation. The activated type I receptor may then phosphorylate the cytoplasmic Smad2 and Smad3 proteins, which then bind into a heteromeric complex with the Smad4 protein. This Smad complex then translocates into the nucleus where it binds to specific DNA sequence motifs to act as a transcription factor, and thereby, it activates expression of target genes including Snail (Masszi et al, 2003; Guarino et al, 2009).

Smads can also combine with the EMTrelated transcription factor lymphoid enhancer factor-1 (LEF-1), which has binding sites for both $\beta$-catenin and Smads, and thereby, they regulate the transcription of genes involved in EMT (Bottinger and Bitzer, 2002). In addition, Smads also induce the expression of ILK, a downstream effector of both TGF- $\beta$-generated and matrix generated signals, which suppresses E-cadherin, up- regulates fibronectin and MMP-2, and promotes EMT through stimulation of the $\beta$ catenin pathway (Choi and Diehli, 2013). Moreover, TGF- $\beta$ signaling underlies cytoskeleton remodeling through the stimulation of the Rho/Rho kinase (ROK) pathway and activation of $\alpha$-smooth muscle actin transcription (Masszi et al, 2003).

b. Extracellular matrix: Extracellular matrix molecules from both basement membrane and interstitium are ligands for the transmembrane receptor family of integrins. Integrin-linked kinase is a cytoplasmic serine-threonine kinase physically interacting with the cytoplasmic domain of $\beta$-integrins and therefore is in the ideal position for transducing integrin-mediated signals from the matrix (Ozaki et al, 2011). In addition, it is also stimulated by growth factor-mediated and cytokine-mediated signals including the TGF- $\beta /$ Smad signaling axis (Wells, 2008). Activated ILK can directly phosphorylate downstream targets such as the kinase Akt and GSK-3 $\beta$, resulting in the inhibition of GSK-3 $\beta$ and subsequent up-regulation of both $\beta$-catenin and Snail (Wells, 2010).

c. Wnt/ $\beta$-catenin: The extracellular Wnt glycoproteins bind to cell surface receptors to stimulate intracytoplasmic events that ultimately lead to inhibition of GSK-3 $\beta$ and, therefore, to cytoplasmic accumulation of $\beta$ catenin (Friedman, 2003). In the absence of a Wnt-mediated signal, most $\beta$-catenin is bound to E-cadherin in junction complexes, whereas the non-bound cytoplasmic amount is rapidly phosphorylated by GSK-3 $\beta$ and thereby targeted for proteasomal degradation. When Wnt signaling is activated and, therefore, GSK-3 $\beta$ is inhibited, $\beta$-catenin accumulates within the cytoplasm and, finally, translocate into the nucleus. These, in combination with the transcription factor LEF-1, activates EMT-related genes, including those encoding fibronectin, vimentin, MMPs, and Snail family members (Guarino et al, 2009). In addition to Wnts, the signaling can be activated by direct stimulation of Akt or ILK that inhibit GSK-3 $\beta$ and similar- 
ly lead to $\beta$-catenin accumulation (Kalluri and Neilson, 2003) as well as to increased Snail. The inhibitory action of GSK-3 $\beta$ on Snail is counteracted by LOXs which, instead, lead to Snail up-regulation (Peinado $e t$ $a l$, 2007). LOXs are activated by hypoxia inducible factor-1, a transcription factor stimulated by hypoxia that has been demonstrated to induce EMT in hypoxic microenvironments including fibrotic lesions (Higgins et al, 2008).

d. Rho GTPases: Most EMT-inducing pathways, including TGF- $\beta$ signaling, influence the activity of the small GTPases of the Rho family are responsible for the regulation of cell shape and motility (Kisseleva and Brenner, 2008). Rho GTPases act as molecular switches by cycling between inactive guanosinediphosphate (GDP)-bound and active guanosine triphosphate (GTP)-bound states and, thereby, regulate the dynamics of the cytoskeleton through the assembly and disassembly of actin-based structures (Jou and Diehl, 2010). Rho activates ROK, a serine-threonine kinase that increases the levels of phospho-myosin thus stimulating actinmyosin filament assembly, and therefore, cellular contractility needed for movement. This enables the most impressive step of EMT, the migration of the transitioning cells from the epithelial into the interstitial compartment (Guarino et al, 2009).

3-Hallmarkers of EMT: The conversion of epithelium into mesenchyme requires alterations in cellular morphology, adhesion, and migratory capacity. A variety of biomarkers have been suggested to define all three subtypes of EMT .These markers include: cellsurface markers as E-cadherin, cytoskeletal markers as Beta catenin and several transcription factors \& microRNAs (Lee et al, 2012).

3.1. cell-surface-markers: Decreased Ecadherin (an epithelial marker) expression is often accompanied by increased expression of $\mathrm{N}$ - cadherin (a mesenchymal marker). Loss of E-cadherin contributes to EMT both by modulating cell-cell adhesion and by al- tering signaling through the sequestration of associated cytoplasmic proteins, including $\beta$-catenin (Peinado et al, 2004; Iwatsuki et al, 2010). E-cadherin maintains cell-cell contacts and epithelial tissue architecture. Decreased expression of E-cadherin has been found in all three types of EMT and is thought to be the prototypical marker of EMT (Kalluri and Neilson, 2003). Studies point to a potentially important mechanism for the enlargement of the resident pool of fibroblasts during the fibrotic reaction of the damaged organ (Guarino et al, 2009). Epithelial cells are adherent cells that closely attach to each other, forming coherent layers in which cells exhibit apical-basal polarity. Mesenchymal cells, in contrast, are nonpolarized cells, capable of moving as individual cells because they lack intercellular connections. EMT describes the process by which cells gradually lose typical epithelial characteristics and acquire mesenchymal traits. MET refers to the reverse process. It is important to emphasize that EMT/MET refer to changes in cell shape and adhesive properties (Lorenzini et al, 2010).

The key epithelial features that are eventually lost during EMT include typical epithelial expression and distribution of proteins that mediate cell-cell and cell-matrix contacts, as well as the cytoskeletal organization that is responsible for normal epithelial polarity (Guarino et al, 2009). The key mesenchymal characteristics that are ultimately gained during EMT include the ability to migrate and invade the surrounding matrix. This migratory/invasive phenotype requires induction of mesenchymal filaments, cytoskeletal rearrangements, and increased production of factors that degrade ECM, as well as new matrix molecules (Bi et al, 2012).

3.2. Cytoskeletal markers: There are several cytoplasmic proteins that are used as markers for EMT. $\beta$-Catenin is an adhesion plaque protein that plays a dual role during EMT. In quiescent epithelium, $\beta$-catenin is located in the cytoplasm and either bound to cadherin or targeted for degradation (Gavert 
and Ben-Ze'ev, 2007). During EMT, $\beta$-catenin translocates into the nucleus and functions as a transcriptional activator together with $\mathrm{T}$ cell factor (TCF/ LEF) complex to regulate the expression of genes associated with EMT including Snail. Nuclear accumulation of $\beta$-catenin has been used as a biomarker for all three types of EMT (Kalluri and Neilson, 2003; Nawshad et al, 2005).

4. Transcriptional factors and microRNAs: The alterations in cellular phenotype do not occur simultaneously. Completion of EMT (or its reversal) requires a carefully-orchestrated series of events that eventually lead to wide-spread changes in gene expression. This is regulated both at the level of gene transcription and via various post-transcriptional mechanisms (Kalluri, 2009). One of the key molecular changes of EMT is depression E-cadherin (Peinado et al, 2007). Several transcriptional repressors of E-cadherin including the zinc-finger protein Snail (Snail/Slug), ZEB (ZEB1/ZEB2), and basic helix-loop-helix factors, such as Twist (Moreno-Bueno et al, 2006). Activation of these transcription factors leads to the repression of E-cadherin, the hallmark of EMT (Kalluri and Neilson, 2003). These proteins function downstream of the EMT-inducing signaling pathways activated by TGF- $\beta$, fibroblast growth factor (FGF), hepatocyte growth factor (HGF), epidermal growth factor (EGF) (Lamouille et al, 2014). Snail has operated as a repressor of E-cadherin. Its expression also increases during liver fibrosis, and its levels correlate with disease progression (Scarpa et al, 2011). In addition to E-cadherin repression, Snail transcription factors contribute to other aspects of EMT. Snail and Slug induce the expression of mesenchymal markers and decrease that of epithelial markers (Kalluri and Neilson, 2003; Lamouille et al, 2014).

MiRNAs are small non-coding RNAs of 18-25 nucleotides that block the translation or induce the degradation of target mRNA. Several miRNAs have been shown to directly target these EMT regulators. MiR-194 is normally expressed in gastrointestinal and liver epithelial cells and has been implicated in EMT regulation. This miRNA directly targets the 3'UTRs of several genes, including mesenchymal marker N-cadherin, Heparin binding EGF, and type 1 insulin-like growth factor, all of which proved to affect the EMT process (Meng et al, 2010). In addition, there is an important functional link between the miR-200 family and ZEB proteins. At the level of transcription, there is double negative-feedback loop between the miR-200 family and ZEB proteins (Burk et al, 2008).

Twist is another key EMT-inducing transcription factor that plays important roles in promoting epithelial motility. Li et al. (2012) found that the expression levels of miR-214 were markedly decreased in metastatic intrahepatic cholangiocarcinoma tissues compared with non-metastatic tissues. Furthermore, inhibition of miR-214, increased transcript levels of Twist, and decreased levels of E-cadherin (Dranoff and Wells, 2010).

MiRNAs can also directly regulate the integrity of epithelial architecture during the EMT process. Ma et al. (2010) found that ectopic expression of miR-9 was able to induce the EMT phenotype, reduce E-cadherin expression, and increase vimentin expression. Another consequence of E-cadherin down-regulation by miR-9 is the activation of $\beta$-catenin signaling; $\beta$-catenin is normally located at the cytoplasmic membrane, and the loss of E-cadherin makes it available for nuclear translocation and E-cadherin expression. MiRNAs can also directly regulate the integrity of epithelial architecture during the EMT process (Lamouille et al, 2014).

\section{Conclusion}

Liver fibrosis is the excessive accumulation of extracellular matrix proteins including collagen occur in nearly all types of chronic liver diseases, which continues as one of the major health problem worldwide. No doubt, liver fibrogenesis is a dynamic and highly integrated molecular, tissue and 
cellular process, potentially reversible, that drives the progression of chronic liver diseases (CLD) towards liver cirrhosis and hepatic failure. The trans-differentiation of epithelial cells into motile mesenchymal cells, a process known as epithelial-mesenchymal transition (EMT), is integral in development, wound healing and stem cell behavior, and contributes pathologically to fibrosis and cancer progression.

\section{Acknowledgements}

The authors would like to thank Dr. Rabab Salem Hamad, the Immunologist, the Central Lab, TBRI, for her kind help and assistance in finalizing the work.

\section{References}

Acloque, H, Admas, MS, Fishwick, K, Bronner-Fraser, M, Nieto, M, 2009: Epithelial-mesenchymal transitions: The importance of changing cell state in development and disease. J. Clin. Invest. 119:1438-7.

Bi, WR, Yang, CQ, Shi, Q., 2012: Transforming growth factor- $\beta 1$ induced epithelial-mesenchymal transition in hepatic fibrosis. Hepatogastroenterol. 59:1960-3.

Bottinger EP, Bitzer M. 2002: TGF-beta signaling in renal disease. J. Am. Soc. Nephrol. 13: 2600-10.

Burk, U, Schubert, J, Wellner, U, Schmalhofer, O, Vincan, E, et al, 2008: A reciprocal repression between ZEB1 and members of the miR-200 family promotes EMT and invasion in cancer cells. EMBO. Rep. 9:582-9.

Choi, S, Diehl, A, 2009: Epithelial-to-mesenchymal transitions in the liver. Hepatol. 50: 2007-13.

Diaz, R, Kim, J, Hui, J, Li, Z, Swain, G, et al, 2008: Evidence for the epithelial to mesenchymal transition in biliary atresia fibrosis. Hum. Pathol. 39:102-15.

Ding, S, Chen, B, 2008: Pathogenesis of hepatic fibrosis analyzed at theproteome level. Saudi Med. J. 33:123-7.

Dranoff, J, Wells, R, 2010: Portal fibroblasts: Underappreciated mediators of biliary fibrosis. Hepatology 51:1438-44.

Friedman, SL, 2003: Liver fibrosis-from bench to bedside. J. Hepatol. 38:S38-53.

Friedman, SL, 2008: Mechanisms of hepatic fibrogenesis. Gastroenterol. 134:1655-69.
Gavert, N, Ben-Ze'ev, A, 2007: b-Catenin signaling in biological control and cancer. J. Cell. Biochem. 102:820-8.

Gressener, AO, Rizk, M, Kovalenko, E, Weiskirchen, R, Gressener, AM, 2008: Changing the pathogenetic roadmap of liver fibrosis? Where did it start; where will it go? J. Gastroenterol. Hepathol. 23:1024-35.

Gressner, AM, Gao, CF, Gressner, OA, 2009: Non-invasive biomarkers formonitoring the fibrogenic process in liver: a short survey. Wld. J. Gastroenterol. 15:2433-40.

Gressner, OA, Gao, CF, 2014: Monitoring fibrogenic progression in the liver. Clin. Chim. Acta. 433:111-22.

Gressner, OA, Weiskirchen, R, Gressner, A, 2007: Evolving concepts of liver fibrogenesis provide new diagnostic and therapeutic options. Comp. Hepatol. 6:7-20.

Guarino, M, Tosoni, A, Nebuloni, I, 2009: Direct contribution of epithelium to organ fibrosis: Epithelial-mesenchymal transition. Hum. Pathol. 40:1365-76.

Hernandez-Gea, V, Friedman, SL, 2011: Pathogenesis of liver fibrosis. Ann. Rev. Pathol. 6: 425-56.

Higgins, DF, Kimura, K, Iwano, M, Haase, V, 2008: Hypoxia-inducible factor signaling in the development of tissue fibrosis. Cell Cycle 7: 1128-32.

Iwatsuki, M, Mimori, K, Yokobori, T, Ishi, H, Beppu, T, et al, 2010: Epithelial-mesenchymal transition in cancer development and its clinical significance. Cancer Sci. 101:293-9.

Jou, J, Diehl, AM, 2010: Epithelial-mesenchymal transitions and hepatocarcinogenesis J. Clin. Invest. 120:1031-4.

Kalluri, R, 2009: EMT: Ehen epithelial cells decide to become mesenchymal-like cells. J. Clin. Invest. 119:1420-8.

Kalluri, R, Neilson, E, 2003: Epithelial-mesenchymal transition and its implications for fibrosis. J. Clin. Invest.112:1776-84.

Keeley, E, Mehrad, B, Streiter, R, 2011: The role of fibrocytes in fibrotic diseases of the lungs and heart. Int. J. Biochem. Cell Biol. 4:2-13.

Kisseleva, T, Brenner, DA, 2008: Fibrogenesis of parenchymal organs. Proc. Am. Thorac. Soc. 5:338-42.

Kisselva, T, Uchinami, H, Feirt, N, Bustamante, OQ, Segovia, JC, et al, 2006: Bone-marrow-derived fibrocytes participate in pathogenesis of liver fibrosis. J. Hepathol. 45:2055-67. 
Kumar, V, Mahato, R, 2015: Delivery and targeting of miRNAs for treating liver fibrosis. Pharm. Res. 32:341-61.

Lamouille, S, Xu, J, Derynck, R, 2014: Molecular mechanisms of epithelial-mesenchymal transition. Nature Rev. Mol. Cell Biol. 15, 3: 178-96.

Lee, KA, Nelson, CM, 2012: New insights into the regulation of epithelial-mesenchymal transition and tissue fibrosis. Int. Rev. Cell Mol. Biol. 294:171-221.

Li, B, Han, Q, Zhu, Y, Yu, Y, Wang, J, et al, 2012: Down-regulation of miR-214 contributes to intrahepatic cholangiocarcinoma metastasis by targeting Twist. FEBS J. 279, 13: 2393-8.

Lo'pez-Novoa, JM, Nieto, MA, 2009: Inflammation and EMT: an alliance towards organ fibrosis and cancer progression. EMBO Mol. Med. 1:303-14.

Lorenzini, S, Bird, T, Boulter, I, 2010: Characterization of a stereotypical cellular and extracellular adult LPC niche in rodents and diseased human liver. Gut 59: 645-54.

Ma, L, Young, J, Prabhala, H, Pan, E, Mestdagh, P, et al, 2010: miR-9, a MYC/MYCN-activated microRNA, regulates E-cadherin and cancer metastasis. Nat. Cell Biol. 12, 3:247-56.

Masszi, A, DiCiano, C, Sirokma'ny, G, Arthur, W, Rotstein, O, et al, 2003: Central role for Rho in TGF-bl-induced a-smooth muscle actin expression during epithelial-mesenchymal transition. Am. J. Physiol. Re. Physiol. 284: F911-24.

Meng, Z, Fu, X, Chen, X, Zeng, S, Tian, Y, et al, 2010: miR-194 is a marker of hepatic epithelial cells and suppresses metastasis of liver cancer cells in mice. Hepatol. 52, 6:2148-57.

Nawshad, A, LaGamba, D, Polad, A, Hay, E, 2005: Transforming Growth Factor- $\beta$ signaling during epithelial-mesenchymal transformation: Implications for embryogenesis and tumor metastasis. Cells Tissues Organs 179:1-23.

Neilson, EG, 2005: Setting a trap for tissue fibrosis. Nat Med; 11:373-4.

Ozaki, I, Hamajima, H, Matsuhashi, S, Mizuta, T, 2011: Regulation of TGF- $\beta 1$-Induced ProApoptotic Signaling by Growth Factor Receptors and Extracellular Matrix Receptor Integrins in the Liver. Front Physiol. 2:1-8.

Peinado, H, Olmeda, D, Cano, A, 2007: Snail, Zeb and bHLH factors in tumour progression: an alliance against the epithelial phenotype? Nat. Rev. Cancer 7:415-28.

Peinado, H, Portillo, F, Cano, A, 2004: Transcriptional regulation of cadherins during development and carcinogenesis. Int. J. Dev. Biol. 48: 365-75.

Pinzani, M, Macias-Barragan, J, 2010: Update on the pathophysiology of liver fibrosis. Expert. Rev. Gastroenterol. Hepatol. 4:459-72.

Roy, S, Benz, F, Luedde, T, Roderburg, C, 2015: The role of miRNAs in the regulation of inflammatory processes during hepatofibrogenesis. Hepatobiliary 4, 1:24-33.

Scarpa, M, Grillo, AR, Brun, P, Macchi, V, Stefani, A, et al, 2011: Snaill transcription factor is a critical mediator of hepatic stellate cell activation following hepatic injury. Am. J. Physiol. Gastrointest. Liver Physiol. 300:G316-26.

Sugimoto, H, Yang, C, LeBleu, V, Soubasakos, M, Giraldo, M, et al. 2007: BMP-7 functions as a novel hormone to facilitate liver regeneration. FASEB J. 21:256-64.

ten Dijke P, Hill, CS, 2004: New insights into TGF-beta-Smad signalling. Trends Biochem, Sci. 29:265-73.

Thiery, JP, Sleeman, JP, 2006: Complex networks orchestrate epithelial-mesenchymal transitions. Nat. Rev. Mol. Cell Biol. 7:131-42.

Wang, S, Hirschberg, R, 2004: Bone morphogenetic protein-7 signals opposing trans-forming growth factor beta $\}$ in mesangial cells. J. Biol. Chem. 279:23200-6.

Wells, RG, 2010: Epithelial to Mesenchymal Transition in Liver Fibrosis: Here Today, Gone Tomorrow? Hepatol. 51, 3:737-40.

Wells, RG, 2008: The role of matrix stiffness in regulating cell behavior. Hepatol. 47:1394-400.

Xue, Z, Wu, X, Liu, M, 2013: Hepatic regeneration and the epithelial to mesenchymal transition. Wld. J. Gastroenterol. 19: 1380-6.

Yook, JI, Li, XY, Ota, I, Hu, C, Kim, HS, et al, 2006: A Wnt-Axin2- GSK3 [beta] cascade regulates Snaill activity in breast cancer cells. Nat. Cell Biol. 8:1398-406

Yusuf, F, Brand-Saberi, B, 2006: The eventful somite: Patterning, fate determination and cell division in the somite. Anat. Embryol. 211:2130.

Zeisberg, M, Neilson, EG, 2009: Biomarkers for epithelial-mesenchymal transitions. J. Clin. Invest.119, 1429-37. 


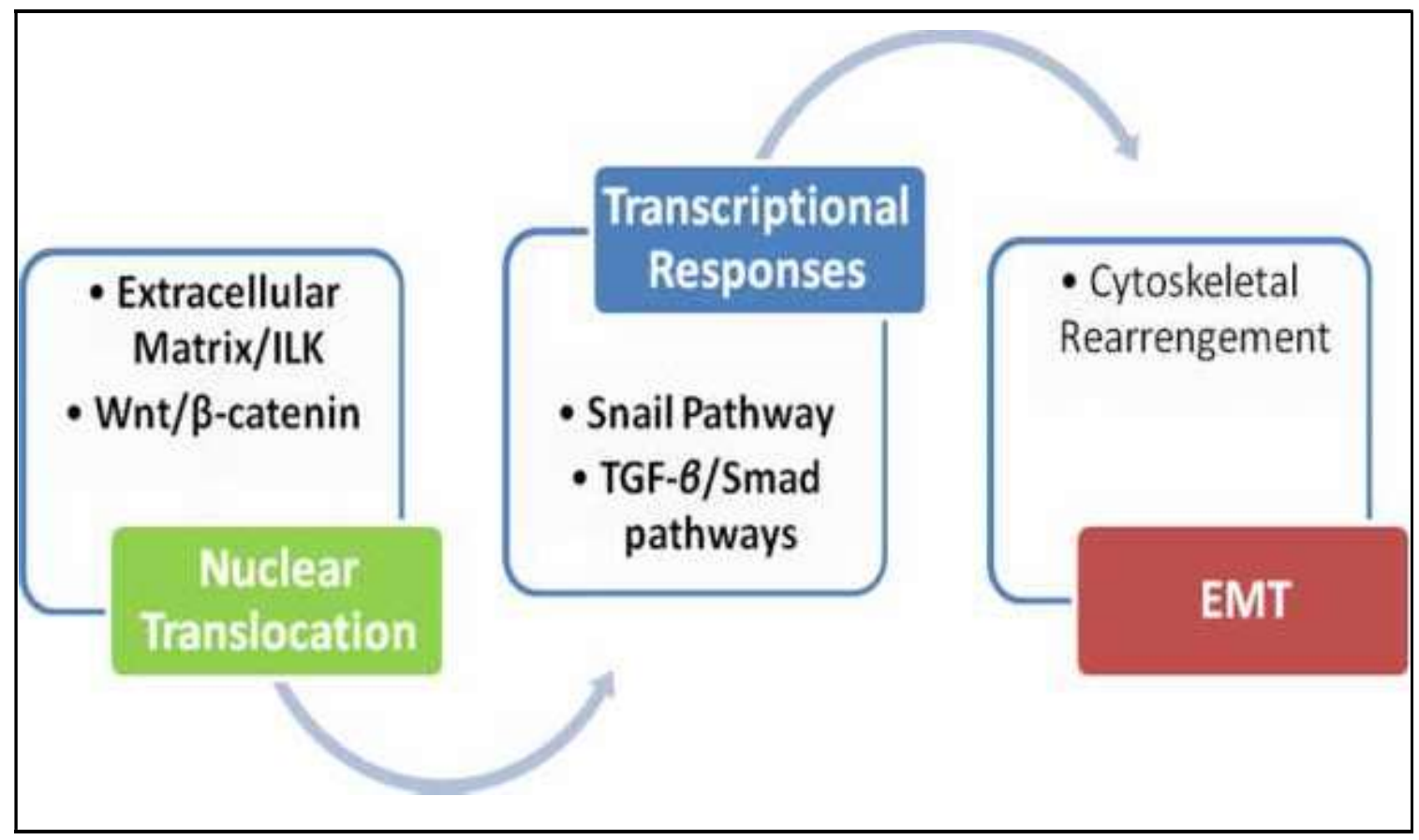

Fig. 1: Simplified representation of main molecular pathways involved in induction of EMT, including Wnt/ $\beta$ catenin, extracellular matrix/ILK, and TGF- $\beta /$ Smad pathways. 